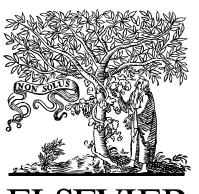

Klotz Communications 2018: Cortisol and all its disorders

\title{
Quality of Life in Cushing's disease: A long term issue?
}

\section{La qualité de vie dans la maladie de Cushing : une affaire à long terme?}

\author{
Susan M. Webb ${ }^{\mathrm{a}, \mathrm{b}, *}$, Alicia Santos ${ }^{\mathrm{a}, \mathrm{b}}$, Eugenia Resmini ${ }^{\mathrm{a}, \mathrm{b}}$, Maria-Antonia Martínez-Momblán ${ }^{\mathrm{b}, \mathrm{c}}$, \\ Luciana Martel $^{\text {a }}$, Elena Valassi ${ }^{\mathrm{a}, \mathrm{b}}$

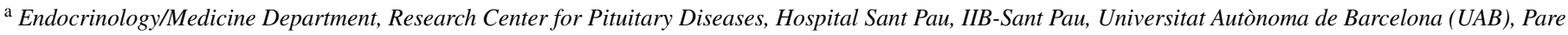 \\ Claret 167, 08025 Barcelona, Spain \\ ${ }^{\mathrm{b}}$ Centro de Investigación Biomédica en Red de Enfermedades Raras (CIBERER, Unidad 747), ISCIII, Spain \\ ${ }^{\mathrm{c}}$ Fundamental and medico-surgical Nursing Department, School of Medicine and Health Sciences, University of Barcelona, L'Hospitalet de Llobregat, Bellvitge \\ Campus, Feixa Llarga, s/n, Pavelló de Govern, 3a planta, despatx 339, 08907 Llobregat, Spain
}

\begin{abstract}
The purpose of this review is to describe how quality of life $(\mathrm{QoL})$ is impaired in patients with hypercortisolism due to Cushing's syndrome of any aetiology, including pituitary-dependent Cushing's disease. It is worse in active disease, but improvement after successful therapy is often incomplete, due to persistent physical and psychological co-morbidities, even years after endocrine "cure". Physical symptoms like extreme fatigability, central obesity with limb atrophy, hypertension, fractures, and different skin abnormalities severely impair the affected patients' everyday life. Psychological and cognitive problems like bad memory, difficulties to concentrate and emotional distress, often associated with anxiety and depression, make it difficult for many patients to overcome the aftermath of treated Cushing's syndrome. Recent studies have shown diffuse structural abnormalities in the central nervous system during active hypercortisolism, thought to be related to the wide distribution of glucocorticoid receptors throughout the brain. Even though they improve after treatment, normalization is often not complete. Shortening the exposure to active Cushing's syndrome by reducing the often long delay to diagnosis and promptly receiving effective treatment is highly desirable, together with preparing the patient for the difficult periods, especially after surgery. In this way they are prepared for the impairments they perceive in every day life, and live with the hope of later improvement, which can be therapeutic in many instances.
\end{abstract}

(C) 2018 Elsevier Masson SAS. All rights reserved.

Keywords: Cushing's syndrome; Quality-of-life impairment; Persistent co-morbidities; Anxiety; Depression; Coping

\section{Résumé}

L'objectif de cette revue est de décrire l'altération de la qualité de la vie en cas d'hypercorticisme induit par la maladie de Cushing, toutes étiologies confondues, y compris le Cushing hypophysaire. Cette altération est maximale en phase active de la maladie, mais l'amélioration apportée par le traitement n'est souvent que partielle, à cause des comorbidités physiques et psychologiques résiduelles, même des années après la « guérison » endocrinologique. Des symptômes physiques tels qu'une fatigabilité extrême, uneobésité centrale avec atrophie musculaire des membres, une hypertension artérielle, des fractures et diverses anomalies cutanées diminuent la qualité de vie quotidienne. Des problèmes psychologiques et cognitifs, tels la perte de mémoire, des difficultés de concentration et la souffrance émotionnelle, souvent associés à l'anxiété et la dépression, empêchent souvent les patients de vaincre les séquelles d'une maladie de Cushing pourtant guérie. Des études récentes rendent compte d'anomalies structurelles diffuses au niveau du système nerveux central en cas de d'hypercorticisme actif, qui seraient dues à la large répartition cérébrale des récepteurs glucocorticoïdes. Ces anomalies sont améliorées par la thérapie, mais le retour au normal est souvent incomplet. Il est donc fortement

\footnotetext{
is Annales d'endocrinologie, 61st International Meeting of Endocrinology H. P. Klotz, Paris from June 21 to June 22, 2018.

* Corresponding author. Dept Endocrinology, Hospital Sant Pau, Pare Claret 167, 08025-Barcelona Spain.

E-mailaddresses: swebb@santpau.cat (S.M.Webb), asantos@santpau.cat (A. Santos), eresmini@santpau.cat (E. Resmini), mmartinezmo46@ub.edu (M.-A. Martínez-Momblán), lmarteld@santpau.cat (L. Martel), evalassi@ santpau.cat (E. Valassi).
} 
souhaitable que la durée d'exposition à la maladie de Cushing active soit réduite, en réduisant le délai diagnostique, qui est souvent long, et en initiant rapidement un traitement adapté, tout en préparant le patient à faire face aux périodes difficiles, notamment au décours de la chirurgie. Ainsi, le patient peut se préparer à une certaine baisse de la qualité de la vie de tous les jours, mais vivre dans l'espoir d'une amélioration ultérieure, espoir qui est souvent lui-même de valeur thérapeutique.

(C) 2018 Elsevier Masson SAS. Tous droits réservés.

Mots clés : Maladie de Cushing ; Perte de qualité de vie ; Comorbidités résiduelles ; Anxiété ; Dépression ; Coping

\section{Introduction}

Hypercortisolism, characteristic of active Cushing's syndrome (CS) determines a host of invalidating signs and symptoms, which negatively impact on patients' health-related quality of life $(\mathrm{QoL})$. These include physical problems like weight gain leading to central obesity, muscle weakness and fatigability, bone fractures, hypertension, skin abnormalities (thinning, easy bruising, red striae, ulcerations) as well as psychological impairment (bad memory, emotional distress, anxiety, depression, etc). Even though surgery with or without additional medical therapy can lead to control of hypercortisolism, often signs and symptoms typical of active hypercortisolism do not revert completely; this is so in Cushing's syndrome (CS) of any aetiology (adrenal, pituitary-dependent Cushing's diseases - CD-, or ectopic ACTH secretion), and determines persistent impairment of QoL, despite being considered endocrinologically "cured" [1].

This prior chronic exposure to endogenous glucocorticoid [GC) excess in CS is also associated with greater morbidity and mortality, related to complications such as components of the metabolic syndrome, cardiovascular events, muscle weakness, bone fractures, neurocognitive impairment and psychiatric disorders [2]. Thus, after resolution of hypercortisolism, patients still often complain of physical and psychological impairment [3], including cognitive changes, depression, less self-confidence and altered illness perception. All this determines a slow and incomplete recovery, severely affecting QoL even long-term after control of cortisol excess [1]. This is a matter of concern for many patients and their partners [4-6] and a challenge for clinicians. To further improve their outcome, patient-reported outcome measures are worth considering, as well as self-management programmes to enhance self-efficacy, as recently shown in a clinical trial [7].

Early detection of hypercortisolism to reduce the delay to diagnosis, and effective treatment aimed at achieving persistent remission is mandatory in CS patients to improve long-term prognosis and QoL [1]. Life-long evaluation with periodic laboratory and clinical assessment, is also highly recommendable even long-term after remission, to appropriately manage comorbidities (diabetes, hypertension, dyslipidemia) according to current guidelines.

\section{The concept of health-related quality of life (QoL)}

QoL is a patient-reported outcome measure, which can be evaluated with generic or disease-generated or specific
Table 1

Psychological phases of adaptation.

1st phase: uncertainty and confusion

2nd phase: bewildered/Negation

3rd phase: opposition/Isolation

4th phase: rage

5th phase: sadness, which leads to either depression, or adaptation (the

latter is easier if you are positive!)

questionnaires. It reflects the individual patient's definition of wellbeing, including perceptions of physical, psychological, emotional and social health issues. Disease-generated questionnaires tend to be more sensitive in identifying limitations specific of the disease, as well as changes after treatment, but cannot be used in normal subjects or in other diseases, while generic questionnaires are useful in any population, and allow comparing QoL in different diseases or with normal subjects. "Domain-specific" questionnaires are designed to evaluate a determined problem, for example, fatigability, pain, dyspnoea or sexual dysfunction, and are therefore useful to compare the degree of impairment of a specific domain [1].

Thus, after psychometric validation and translation into the languages of the patient populations to be investigated, QoL scores can be used to highlight the patient's impression on clinical aspects often not approached clinically, despite being important for everyday life [8], and complement the biochemical and radiological tests routinely used in patient's follow-up.

\section{Effect o chronic hypercortisolism on health-related quality of life}

With the availability of two different Cushing-specific QoL questionnaires (CushingQoL [9] and Tuebingen CD-25 [10,11]), new knowledge on impairment suffered by CS patients has emerged. CushingQoL consists of 12 questions with a five option Likert scale answer, which was described as unidimensional [12]. Recently, however, two subscales (psychosocial issues and physical problems) were identified and showed adequate model fit. Data were obtained from patients in remission from CS $(n=341)$ recruited from the Cushing's Syndrome Research Foundation's email lists; they completed the CushingQoL questionnaire and a short demographics survey [13]. Regardless of the scoring solution used, it was concluded that CushingQoL had proven to be a valuable resource for assessing health-related QoL in patients with CS (Table 1).

The Tuebingen CD quality of life inventory (Tuebingen CD25) $[10,11]$ is a 25 -item questionnaire covering six essential 
domains of HRQoL. It has showed improvement after successful surgery in $17 \mathrm{CD}$ patients [14]; three quarters of the patients had impaired QoL preoperatively, but only one third after successful surgery. A better postoperative QoL was observed in the presence of less comorbidities $(\leq 2)$ and greater morning cortisol decrease. Predictors for postoperative improvement in QoL were greater preoperative QoL impairment and a younger age, as also reported using the CushingQoL questionnaire [15]. Other predictors of impaired QoL are having experienced a long delay until a correct diagnosis of CS was obtained [16] or having seen more physicians before a correct diagnosis of CS was made $[5,17]$. Whether this means that patients who experience greater discomfort demand more physician visits, or it simply indicates a longer duration of symptoms, is not yet clear. In comparison, patients who are treated by physicians who specialize in CS report better QoL. Since a bad QoL can influence working capacity, it may have major economic consequences for patients and their families [5,17].

Similar results with the SF-36 and Tuebingen CD-25 questionnaires were also reported after bilateral adrenalectomy in 50 patients with CS, most of which were followed for a median of 11 years [18]. While comorbidities like hypertension, diabetes mellitus, osteoporosis, muscle weakness, cushingoid phenotype and menstrual irregularity improved after surgery, QoL was still impaired in $45 \%$ of female and $16.7 \%$ of male patients, when compared with a healthy population [18].

\subsection{Physical limitations in Cushing's syndrome}

Endogenous (of adrenal, pituitary or ectopic origin) or exogenous CS (due to treatment with GC) is associated with persistent physical morbidity (including increased cardiovascular risk, fatigability, myopathy and bone fragility) which negatively impact on well-being and QoL. This QoL impairment has been shown with both generic and dimension-specific questionnaires (i.e., SF-36 survey, the Hospital Anxiety and Depression Scale (HADS)), and disease-specific questionnaires, like the CushingQoL [19-21] and the Tuebingen CD-25 questionnaires [14].

The characteristic physical complaints of active CS impair QoL. Data from the European Cushing syndrome registry ERCUSYN show that weight gain is reported in over $80 \%$ of patients, hypertension, skin alterations and myopathy in at least two thirds, and other complaints in descending order of frequency are hirsutism, menstrual irregularities, reduced libido, diabetes mellitus and fractures (the latter observed in at least $20 \%$ of the patients). The negative impact on QoL was further increased by the delay to diagnosis of a median of two years (interquartile range: three years) in the overall series [15]. And even after surgery, the time to see an improvement in symptoms and signs like hypertension, menstrual irregularity, moon face and plethora, central obesity, purple striae or easy bruising is over 6 months on average, and 9 to 12 months for buffalo hump, hirsutism, diabetes and muscle weakness [22].

Although obesity is a characteristic of CS, differences in QoL were identified between both diseases, using the SF-36 survey and a symptom questionnaire [23]. Obese patients, mostly women with a mean average age between 40 and 50 years, had better QoL in the mean physical component summary (PCS) score, but worse mean mental component summary (MCS) than CS patients. The presence of more CS symptoms worsened the PCS and MCS scores, while obese patients surprisingly showed worse mental health scores than the CS population. BMI correlated with PCS in obesity, but not in CS, and was not associated with MCS in either group.

A large study of 123 patients with CS of both pituitary and adrenal origin in remission for a mean of $13.3+10.4$ years, showed worse QoL on practically all dimensions investigated, compared to a matched control group. If patients were in remission after pituitary surgery without hormonal deficiencies, QoL was impaired in only $50 \%$ of the dimensions evaluated. A shorter duration of remission and women (compared to men) had a negative impact on QoL, and it remained impaired despite long-term remission of CS, independently of aetiology (i.e., pituitary, adrenal, or ectopic ACTH secretion), the presence or not of hormonal deficiencies and treatment strategies, pointing to hypercortisolism as the cause of these persistent problems, rather than the location of the originating tumour [17,24].

\subsection{Psychological consequences of Cushing's syndrome}

Hypercortisolism exerts irreversible toxicity on brain structures and determines sustained psychological dysfunction and structural brain abnormalities, including atrophy of different structures, even after remission of hypercortisolism [25,26]. These brain volume decreases are especially seen in active hypercortisolism and in patients diagnosed at an older age, which is thought to be related to less brain plasticity compared to younger subjects; other factors like longer disease duration and longer exposure to hypercortisolism also are related to worse psychological outcome [25-27] (Fig. 1).

Using the Beck Depression Inventory II (BDI-II), the SF36 and the multidimensional body-self relations questionnaire (MBSRQ), QoL in the general health category and perception of body image were worse in 40 patients with $\mathrm{CD}$ who underwent transsphenoidal surgery, compared to 40 healthy controls [28]. In contrast with several other studies, however, no differences in depression scores were found between CD patients globally and controls. But in CD patients without remission, depression was more prevalent than in both $\mathrm{CD}$ patients with remission and healthy subjects. Additionally, physical functioning, bodily pain and general health scores of the SF-36 questionnaire in CD patients without remission were also worse than in CD patients in remission and healthy subjects.

A higher prevalence of "maladaptive" personality traits in CS patients even after long-term cure, may affect their everyday QoL [29]. They appear to perceive that they are characterized by "unpleasant" increased-anxiety traits (pessimism, neuroticism, shyness with strangers and low extraversion), and try to answer in a rather "pleasant" (or socially desirable) way.

\section{Changes in QoL over time in CS}

Even though after successful treatment some improvement is usually perceived, it often takes months or over a year, so that the 

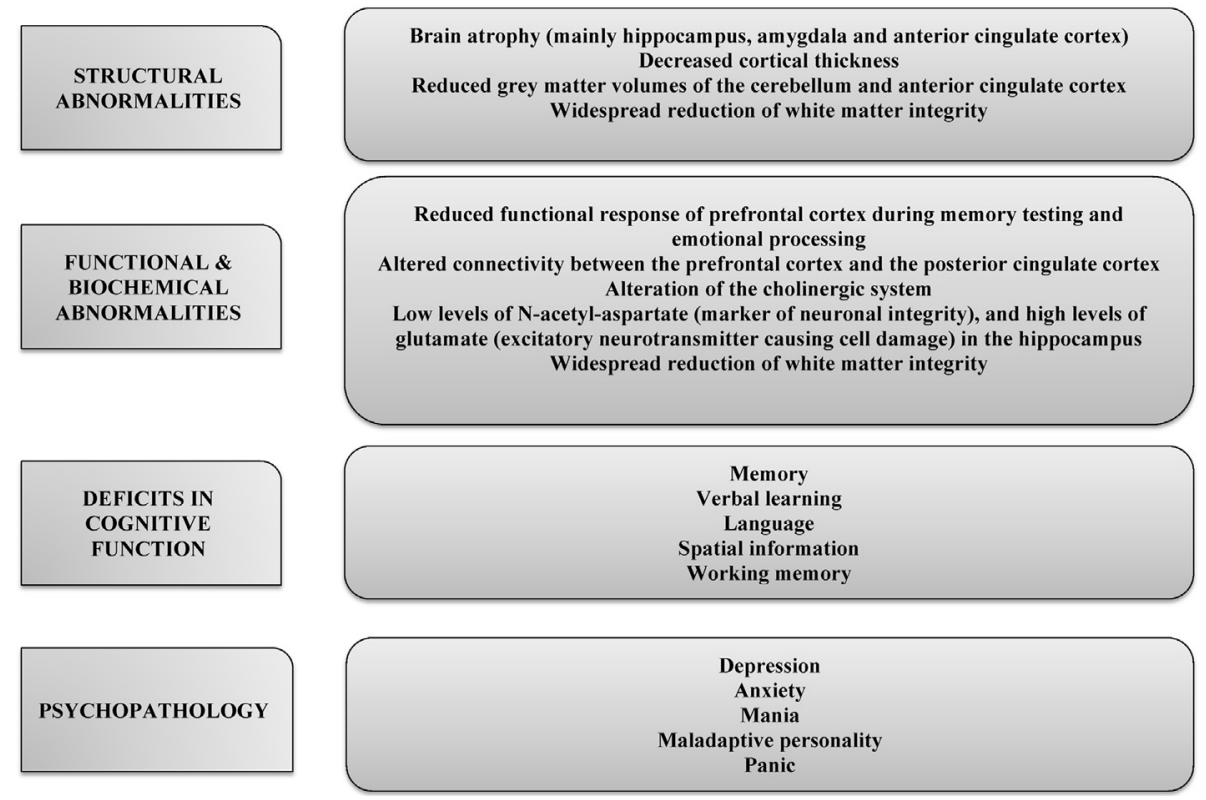

Fig. 1. Structural brain abnormalities and neuropsychiatric disorders described in Cushing's syndrome [25].

immediate postoperative phase can be very challenging due to a "glucocorticoid deprivation phase", characterised by more pain, severe fatigability, worries about physical appearance, troubles with their family life and partner relations, decreased work or school performance, helplessness often leading to depression and a bad perception of QoL [5]. Additionally, attempting to find the lowest glucocorticoid substitution dose possible to maintain a satisfactory well-being after successful surgery (leading to adrenal axis inhibition), and favour endogenous recovery of the adrenal axis inhibition, may be difficult.

Predictors for worse QoL in CS include a long delay to diagnosis of CS [30], requiring more physicians before a correct diagnosis [31,32], and presence of 'maladaptive' personality traits like pessimism, neuroticism, shyness with strangers and low extraversion [33]. In contrast, a worse baseline QoL score, a younger age, and being treated by specialists knowledgeable in CS predict a greater postoperative improvement in QoL [34].

Making the patients aware of the psychological and physical consequences of successful treatment and therefore being "cured" helps to understand this difficult period for the patients. It is important to prepare the patient so he/she is aware and can try to cope with the aftermath, a first step to facilitate improvement in the long term, since they tend to experience persistently negative illness perceptions. Most patients don't feel much better after treatment, and some even feel worse; this is most frequently related to sustained fatigue, decreased muscle strength and weakness, weight gain especially around the abdomen, forgetfulness, trouble sleeping, depression, anxiety, and poor psychosocial functioning [5], consistent with long-lasting negative effects of previous hypercortisolism on cognitive function and behaviour $[17,18]$. All this negatively influences QoL, even after more than 10 years of remission [35,36]. Additionally, duration of adrenal insufficiency and hydrocortisone usage are inversely associated with QoL in treated CS, irrespective of aetiology $[12,13,24]$.
Another longitudinal study in 17 patients with active hypercortisolism, investigated the effect of sequential stepwise medical therapy on QoL and recovery of physiological salivary and plasma cortisol diurnal rhythm [37]. Treatment with the somatostatin analogue pasireotide was followed by the dopamine agonist cabergoline, and later the adrenal-blocking agent ketoconazole if normal urinary free cortisol (UFC) was not reached. The CushingQoL, Nottingham Health Profile (NHP), HADS, Multidimensional Fatigue Inventory (MFI) and RAND36 instruments were evaluated. RAND-36 includes the same set of items as SF-36, and were both developed in the Medical Outcomes Study, but scoring of general health and pain scales differ [38]. After 80 days, $88 \%$ (15/17) of the patients normalized UFC. Patients then either continued medical therapy for 1 year or underwent surgery. Cortisol diurnal rhythm was preserved at baseline in 5/17 patients; in another 6/12 with disturbed baseline rhythm, recovery occurred, but not correlated with QoL, which was impaired in most subscales (18/20) in CD patients compared to literature-derived controls. After 80 days, patients reported more pain (interpreted as a reflection of steroid-withdrawal), but QoL did not improve or deteriorate. Baseline CushingQoL scores (around 50) tended to improve after 1 year of remission in 3 patients that continued medical therapy (to over 75), reflecting how long it takes for QoL to recover, despite control of hypercortisolism.

A phase III, randomized, double-blind study assessed the relationships between QoL and UFC and CD-related signs and symptoms in patients with elevated UFC receiving s.c. pasireotide 600 or $900 \mathrm{mg}$ twice daily for 12 months [21,39]. Baseline QoL using CushingQoL, clinical signs and features of $\mathrm{CD}$ and depression (evaluated with the Beck Depression Inventory II (BDI-II)) were compared to follow-up evaluation. Changes in CushingQoL and the proportion of patients achieving a clinically meaningful improvement in CushingQoL were also compared among patients stratified by mean UFC con- 
Table 2

The example of psychological adaptation in Cushing's syndrome.

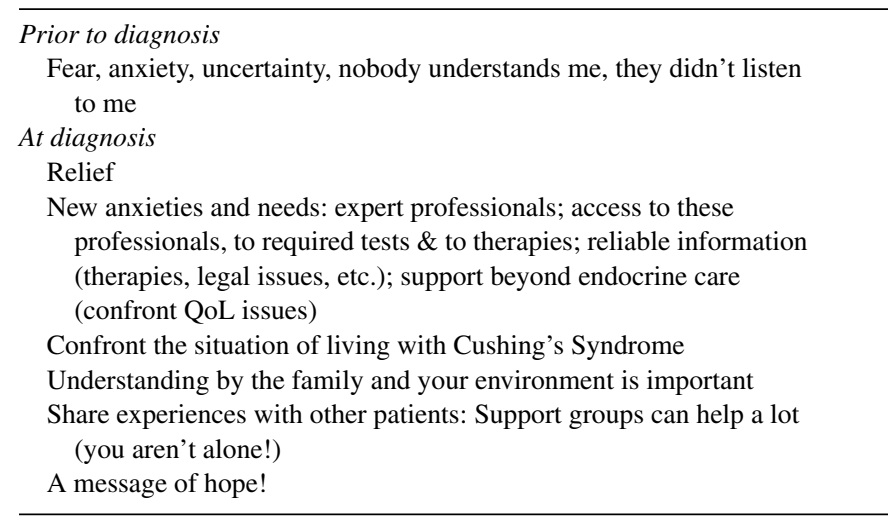

trol status (controlled, partially controlled, and uncontrolled) at month 6. Analyses were also conducted at month 12, with multivariable adjustment. Change in CushingQoL was correlated with changes in mean UFC, BMI, weight, and BDI-II at month 12 , but not at month 6. CushingQoL scores at month 12 were better for controlled and partially controlled patients than for uncontrolled patients with persistently elevated UFC. This led to conclude that among patients achieving biochemical control with daily s.c pasireotide treatment, improvement of QoL was observed at 12 months, especially in patients reaching biochemical control [39]. The minimal important difference in CushingQoL score to be clinically meaningful was found to be 10.1, based on the half-standard deviation distribution method [21] as recommended by the FDA and other regulatory agencies in the USA and Europe, allowing comparison of different treatments on the patient's outcome. This longitudinal study confirmed CushingQoL as a reliable, valid, and responsive (good test-retest reliability and sensitivity to change) questionnaire to assess QoL and other relevant clinical outcomes in adults with CD (Table 2).

\section{How can patients attempt to improve QoL after being diagnosed with CS}

Even in the long-term management of CS, being aware of the changing physical and psychological situations does help patients conform, with the hope of final improvement [40]. One should be aware that common complaints like cognitive impairments and psychiatric disorders, as well as periodic follow-ups to evaluate pituitary function and detect possible recurrences of hypercortisolism, all put extra stress on these CS patients, which in turn worsens psychological issues and often leads to a bad QoL. Encouraging the patients to take care of themselves can help them feel better and improve their QoL, with the support of friends and family, so they can feel they are taking the reins of their lives again. Things to recommend are to consider taking up new hobbies or challenges within their capacities, as well as following healthy diets and regular exercise and taking their prescribed treatment as indicated [31]. It is worth transmitting a message of hope, in the sense that despite the many
Table 3

Significant improvements obtained with a nursing educational program on Cushing's syndrome patients compared to control patients followed routinely [41].

Greater physical activity

Better adherence to therapy

Quantitative and qualitative improvement in sleep patterns

Less pain

Reduction of health costs

Number of smoked cigarettes

Positive correlation between pain reduction and quality of life improvement

changes after CS, it is still possible to be happy if one works at it [17].

A specific nursing educational program, addressed to $\mathrm{CS}$ patients has been shown to exert a positive effect on different living habits and in parallel, improved QoL. They improved their physical activity, healthy lifestyle habits, adherence to therapy, sleep patterns and experienced a reduction in pain level and in the use of health resources, all influencing QoL positively. Those patients who improved most with this educational intervention were those with greatest impairment in their QoL [41], while if the patient was depressed, recovery of QoL was more difficult (Table 3).

\section{Conclusions}

Over the last decade it has become evident that QoL is severely affected in patients diagnosed with Cushing' syndrome, and may not normalize despite successful treatment and endocrine "cure". The reasons are multifactorial, including physical symptoms (including fatigability, abnormal body image due to central obesity and several skin problems, hypertension, etc) and psychological complaints; additionally, morphological abnormalities in the central nervous system, reflect the glucocorticoid toxicity on the brain, presumably through the glucocorticoid receptors identified in different brain areas.

Understanding and encouragement by the health care providers, and in some cases psychological support can help patients through this difficult period with the hope of ultimate improvement.

\section{Disclosure of interest}

The authors declare that they have no competing interest.

\section{References}

[1] Webb SM, Crespo I, Santos A, Resmini E, Aulinas A, Valassi E. Quality of life tools for the management of pituitary disease. Eur J Endocrinol 2017; 177:R13-26.

[2] Newell-Price J, Bertagna X, Grossman AB, Nieman LK. Cushing's syndrome. Lancet 2006;367:1605-17.

[3] Valassi E, Crespo I, Santos A, Webb SM. Clinical consequences of Cushing's syndrome. Pituitary 2012;15:319-29.

[4] Santos A, Resmini E, Pascual JC, Crespo I, Webb SM. Psychiatric symptoms in patients with cushing's syndrome: prevalence, diagnosis and management. Drugs 2017, http://dx.doi.org/10.1007/s40265-017-0735-z. 
[5] Gotch PM. Cushing's syndrome from the patient perspective. Endocrinol Metab Clin North Am 1994;23:607-17.

[6] Andela CD, Scharloo M, Ramondt S, Tiemensma J, Husson O, Llahana S, et al. The development and validation of the Leiden Bother and needs questionnaire for patients with pituitary disease: the LBNQ-Pituitary. Pituitary 2016;19:293-302.

[7] Andela CD, Repping-Wuts H, Stikkelbroeck NMML, Pronk MC, Tiemensma J, Hermus AR, et al. Enhanced self-efficacy after a selfmanagement programme in pituitary disease: a randomized controlled trial. Eur J Endocrinol 2017;177:59-72.

[8] Schipper H, Clinch JJ, Olweny CLM. Quality of life studies: definitions and conceptual issues. In: Spilker B, editor. Quality of life assessments in clinical trials. New York: Raven Press Ltd; 1990. p. 16.

[9] Santos A, Resmini E, Martínez-Momblán MA, Crespo I, Valassi E, Roset $\mathrm{M}$, et al. Psychometric performance of the CushingQoL questionnaire in conditions of real clinical practice. Eur J Endocrinol 2012;167:337-42.

[10] Milian M, Teufel P, Honegger J, Gallwitz B, Schnauder G, Psaras T. The development of the Tuebingen Cushing's disease quality of life inventory (Tuebingen CD-25). Part I: construction and psychometric properties. Clin Endocrinol (Oxf) 2012;76:851-60.

[11] Milian M, Teufel P, Honegger J, Gallwitz B, Schnauder G, Psaras T:. The development of the Tuebingen Cushing's disease quality of life inventory (Tuebingen CD-25). Part II: normative data from 1,784 healthy people. Clin Endocrinol (Oxf) 2012;76:861-7.

[12] Webb SM, Badia X, Barahona MJ, Colao A, Strasburger CJ, Tabarin A, et al. Evaluation of health-related quality of life in patients with Cushing's syndrome with a new questionnaire. Eur J Endocrinol 2008;158:623-30.

[13] Tiemensma J, Depaoli S, Felt JM. Using subscales when scoring the Cushing's quality of life questionnaire. Eur J Endocrinol 2016;174:33-40.

[14] Milian M, Honegger J, Teufel P, Wolf A, Psaras T. Tuebingen CD-25 is a sensitive tool to investigate health-related quality of life in Cushing's disease patients in the course of the disease. Neuroendocrinol 2013;98:188-99.

[15] Valassi E, Santos A, Yaneva M, Tóth M, Strasburger CJ, Chanson P, et al. The European Registry on Cushing's syndrome: 2-year experience. Baseline demographic and clinical characteristics. Eur J Endocrinol 2011;165:383-92.

[16] Valassi E, Crespo I, Keevil BG, Aulinas A, Urgell E, Santos A, et al. Affective alterations in patients with Cushing's syndrome in remission are associated with decreased BDNF and cortisone levels. Eur J Endocrinol 2017; 176:221-31

[17] Santos A, Webb SM. Coping with Cushing's syndrome. The patients' perspectives. In: Laws Jr ER, editor. Cushing's disease an often misdiagnosed and not so rare disorder. Amsterdam: Elsevier Academic Press; 2017. p. $170-85$.

[18] Curto L, Ferrau F, Trimarchi F. Health-related quality of life in patients with Cushing's syndrome. Minerva Endocrinol 2014;39:75-7.

[19] Badia X, Roset M, Valassi E, Franz H, Forsythe A, Webb SM. Mapping CushingQoL scores to EQ-5D utility values using data from the European Registry on Cushing's syndrome (ERCUSYN). Qual Life Res 2013;22:2941-50.

[20] Roset M, Badia B, Forsythe A, Webb SM, CushingQoL Development Group. Mapping CushingQoL scores onto SF-6D utility values in patients with Cushing's syndrome. Patient 2013;6:103-11.

[21] Nelson LM, Forsythe A, McLeod L, Pulgar S, Maldonado M, Coles T, et al. Psychometric evaluation of the Cushing's Quality-of-Life questionnaire. Patient 2013;6:113-24.

[22] Sippel RS, Elaraj DM, Kebebew E, Lindsay S, Tyrrell JB, Duh QY. Waiting for change: symptom resolution after adrenalectomy for Cushing's syndrome. Surgery 2008;144:1054-61.

[23] Abraham SB, Abel BS, Rubino D, Nansel T, Ramsey S, Nieman LK. A direct comparison of quality of life in obese and Cushing's syndrome patients. Eur J Endocrinol 2013;168:787-93.
[24] Wagenmakers MA, Netea-Maier RT, Prins JB, Dekkers T, den Heijer M, Hermus AR. Impaired quality of life in patients in long-term remission of Cushing's syndrome of both adrenal and pituitary origin: a remaining effect of long-standing hypercortisolism? Eur J Endocrinol 2012;167: 687-95.

[25] Andela CD, van Haalen FM, Ragnarsson O, Papakokkinou E, Johannsson G, Santos A, et al. Mechanisms in endocrinology: Cushing's syndrome causes irreversible effects on the human brain: a systematic review of structural and functional magnetic resonance imaging studies. Eur J Endocrinol 2015;73:R1-14.

[26] Santos A, Resmini E, Crespo I, Pires P, Vives-Gilabert Y, Granell E, et al Small cerebellar cortex volume in patients with active Cushing's syndrome. Eur J Endocrinol 2014;171:461-9.

[27] Resmini E, Santos A, Gómez-Ansón B, Vives Y, Pires P, Crespo I, et al Verbal and visual memory performance and hippocampal volumes, measured by 3-Tesla magnetic resonance imaging, in patients with Cushing's syndrome. J Clin Endocrinol Metab 2012;97:663-71.

[28] Alcalar N, Ozkan S, Kadioglu P, Celik O, Cagatay P, Kucukyuruk B, et al. Evaluation of depression, quality of life and body image in patients with Cushing's disease. Pituitary 2013;16:333-40.

[29] Tiemensma J, Biermasz NR, Middelkoop HA, van der Mast RC, Romijn JA, Pereira AM. Increased prevalence of psychopathology and maladaptive personality traits after long-term cure of Cushing's disease. J Clin Endocrinol Metab 2010;95:E129-41.

[30] Cohen SI. Cushing's syndrome: a psychiatric study of 29 patients. Br J Psychiatry 1980;136:120-4.

[31] Dorn LD, Burgess ES, Friedman TC, Dubbert B, Gold PW, Chrousos GP. The longitudinal course of psychopathology in Cushing's syndrome after correction of hypercortisolism. J Clin Endocrinol Metab 1997;82: 912-9.

[32] Starkman MN, Giordani B, Gebarski SS, Schteingart DE. Improvement in mood and ideation associated with increase in right caudate volume. J Affect Disord 2007;101:139-47.

[33] Jeffcoate WJ, Silverstone JT, Edwards CR, Besser GM. Psychiatric manifestations of Cushing's syndrome: response to lowering of plasma cortisol. Q J Med 1979;48:465-72.

[34] Ragnarsson O, Berglund P, Eder DN, Johannsson G. Long-term cognitive impairments and attentional deficits in patients with Cushing's disease and cortisol-producing adrenal adenoma in remission. J Clin Endocrinol Metab 2012;97:1640-8.

[35] Tiemensma J, Kaptein AA, Pereira AM, Smit JW, Romijn JA, Biermasz NR. Negative illness perceptions are associated with impaired quality of life in patients after long-term remission of Cushing's syndrome. Eur J Endocrinol 2011;165:527-35.

[36] Tiemensma J, Kaptein AA, Pereira AM, Smit JW, Romijn JA, Biermasz NR. Coping strategies in patients after treatment for functioning or nonfunctioning pituitary adenomas. J Clin Endocrinol Metab 2011;96:964-71.

[37] Feelders RA, de Bruin C, Pereira AM, Romijn JA, Netea-Maier RT, Hermus AR, Pierre M, Zelissen, Ramona van Heerebeek, et al. Pasireotide alone or with cabergoline and ketoconazole in Cushing's disease. N Engl J Med 2010;362:1846-8.

[38] Hays RD, Sherbourne CD, Mazel RM. The RAND 36-Item health survey 1.0. Health Econ 1993;2:217-27.

[39] Webb SM, Ware JE, Forsythe A, Yang M, Badia X, Nelson LM, et al. Treatment effectiveness of pasireotide on health-related quality of life in patients with Cushing's disease. Eur J Endocrinol 2014;171: 89-98.

[40] Pereira AM, Tiemensma J, Romijn JA. Neuropsychiatric disorders in Cushing's syndrome. Neuroendocrinol 2010;92:65-70.

[41] Martínez-Momblán MA, Gómez C, Santos A, Porta N, Esteve J, Úbeda I, et al. A specific nursing educational program in patients with Cushing's syndrome. Endocrine 2016;53:199-209. 